International Journal of Physical Research, $9(1)(2021) 11-26$
International Journal of Physical Research
SPC
Website: www.sciencepubco.com/index.php/IJPR
Research paper

\title{
New closed-form soliton solutions for the tzitzeica dodd bullough equation arising nonlinear optics via three distinct re-liable approaches
}

\author{
Faisal Hawlader ${ }^{1}$, Nahida Akter ${ }^{1 *}$ \\ ${ }^{1}$ Department of Mathematics, Bangabandhu Sheikh Mujibur Rahman Science and Technology University, Gopalganj-8100, Bangladesh \\ *Corresponding author E-mail: nahida.bsmrstu18@gmail.com
}

\begin{abstract}
Tzitzeica Dodd Bullough (TDB) equation appears in the field of quantum field theory and nonlinear optics. In this article, we extracted abundant new soliton solutions with free choice of arbitrary parameters to the Tzitzeica-Dodd-Bullough (TDB) equation through the three separate methods such as the enhanced -expansion method, the improved -expansion method and the -expansion method by means of the wave transformation and the Painleve property. In these schemes, we formally derived some new closed form soliton solutions of the TDB equation through with symbolic computation package Maple. Soliton solutions are expressed by hyperbolic function, trigonometric function and rational function. The attained solutions are verified by symbolic computation software Maple 17. The attained solutions can be demonstrated by two-dimensional (2D) and three-dimensional (3D) graphs. Finally, it can be concluded that the adopted methods are very effective and well-suited to find new closed-form soliton solutions to the other nonlinear evaluation equations (NLEEs) with integer or fractional order.
\end{abstract}

Keywords: Tzitzeica-Dodd-Bullough Equation; Enhanced -Expansion Method; Improved-Expansion Method; - Expansion Method; Closed-Form Soliton Solution.

\section{Introduction}

Nonlinear evolution equations survive naturally everywhere in the world in various fields of applied mathematics, mathematical physics and engineering, especially plasma physics, solid state physics, plasma waves, chemical kinematics, optical fibers, fluid dynamics and etc. Nonlinear evolution equations (NLEEs) have a great importance to mark out complex physical phenomena in the real-world problems. Therefore, the study of solutions to NLEEs is very much important part to analyze the physical interpretations. It is a fact that, exact solutions provide much physical information and help one to understand the mechanism that governs some physical models, the numerical, analytical and semi-analytical treatments are involved in this regard. An analytical solution is preferred because one can get some insight on the physical phenomena and behaviors from it. So, it is well recognized that to looking for exact analytical solution of NLEEs arising in mathematical physics plays an imperative role in the study of nonlinear physical phenomena and optics. Moreover, the solutions of NLEEs are significantly important in field of soliton theory [1-3]. Nowadays, it is very easier to extract the exact analytical solutions for researcher due to rapid growth of computer algebra based symbolic computation software such as Maple, MATLAB, and Mathematica.

Therefore, researchers have made a perform to ongoing research works and produce the new exact solitary wave solutions from nonlinear evolution equations. Accordingly, they developed numerous potential and useful techniques, such as, the homogeneous balance method [4], Hirota's bilinear method [5], the trial function method [6], the tanh-function method [7], the theta function method [8,9], the extended tanh-function method [10], the modified extended tanh-function method [11], the hyperbolic function method [12], the sine-cosine method [13], the inverse scattering transform [14], the Jacobi elliptic function expansion [15], the Homotopy perturbation methods [16], the auxiliary equation method [17], the first integral method [18], the modified Kudryashov method [19], the generalized Kudryashov method [20], the $\left(G^{\prime} / G\right)$-expansion method [21], the improved $\left(G^{\prime} / G\right)$-expansion method [22] and so on. Practically, there is no integrated fixed method that can be applied to handle all types of nonlinear problems. After that, many researchers tried to propose and execute distinct new method for acquiring new solutions for new nonlinear evolution equations. For further enquiry, we will apply three imperative methods likely the enhanced $\left(G^{\prime} / G\right)$-expansion method [23], the improved $\left(1 / G^{\prime}\right)$-expansion method [24, 25] and the $\left(G^{\prime} / G, 1 / G\right)$-expansion method [26, 27], to examine closed form solution for NLEEs. Essentially, these ameliorate three methods can be thought as an extension of the basic $\left(G^{\prime} / G\right)$-expansion method [21]. The key plan of this article is to acquire the newer and general solutions of the TDB equation with different pattern.

Recently, Wazwaz [28] investigated the nonlinear Zhiber- Shabbat equation: 
$u_{x t}+p e^{u}+q e^{-u}+r e^{-2 u}=0$

Where $p, q$ and $r$ are arbitrary constants. For choosing arbitrary constant parameters, if $q=r=0$, Eq. (1) reduces to the Liouville equation. For $q=0, r \neq 0$, Eq. (1) gives the well-known Dodd-Bullough-Mikhailov (DBM) equation and for $q \neq 0, r=0$, Eq. (1) reduces to the sinh-Gordon equation. Moreover, for $p=0, q=-1, r=-1$, we obtained the Tzitzeica- Dodd- Bullough (TDB) equation as follows [28]:

$u_{x t}-e^{-u}-e^{-2 u}=0$

Equation (2) plays an imperative role in many scientific applications arising in nonlinear optics, plasma physics and quantum field theory [28-44]. Several researchers obtained the different types of exact solutions of the Tzitzeica-Dodd-Bullough equation by applying numerous analytic methods such as, the tanh method [28], [29], the modified simple equation method [30], the bifurcation method [31], the modified tanh-coth function method [32], the $\left(G^{\prime} / G\right)$-expansion method [33,34], the improved tan(\$( $\left.\left.\$\right) / 2\right)$-expansion method [35],

the $\exp (-\Phi(\xi))$-expansion method [36], the modified Kudryashov method [37], an ansatz approach with the Jacobi elliptic function method [38], the exponential rational function method [39], [40], the exp and double exp function method [41], [42], the direct algebraic method [43], the novel exponential rational function method [44], the sine-Gordon expansion method [45] and the modified version of the improved $\tan (\Phi(\xi) / 2)$-expansion method [46]. Interested reader can read the other references article herein [47], [48]. However, no one studied the solutions to the aforesaid equation through the enhanced $\left(G^{\prime} / G\right)$-expansion method, the improved $\left(1 / G^{\prime}\right)$-expansion method and the $\left(G^{\prime} / G, 1 / G\right)$-expansion method. In this article, we will employ first time, the enhanced $(G$ '/ $G)$-expansion method, the improved $\left(1 / G^{\prime}\right)$-expansion method and the $\left(G^{\prime} / G, 1 / G\right)$-expansion method to examine the closed form solitary wave solutions to the

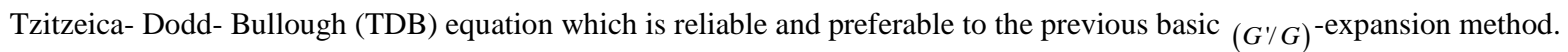

Therefore, the objective of this study is to employ the enhanced $\left(G^{\prime} / G\right)$-expansion method, the improved $\left(1 / G^{\prime}\right)$-expansion method and the $\left(G^{\prime} / G, 1 / G\right)$-expansion method for generating the more general and newer exact solutions of the Tzitzeica-Dodd-Bullough (TBD) equation.

The remainder of the paper is arranged as follows. In section 2, we describe the methodology of the three working methods. In section 3 , we apply these methods to solve the nonlinear evaluation equations point out above. In section 4 and 5 , we illustrate these methods in detail with TDB equation. Finally, we decorate a concluding remark in last section.

\section{A brief description of the solution procedure}

In this section, we will elaborate the algorithm of the enhanced $\left(G^{\prime} / G\right)$-expansion method, the improved $\left(1 / G^{\prime}\right)$-expansion and the $\left(G^{\prime} / G, 1 / G\right)$-expansion method for investigating exact solutions of nonlinear evolution equations (NLEEs). Suppose we have NLEEs for $u(x, t)$, in the form

$P\left(u, u_{t}, u_{x}, u_{x x}, u_{x t}, u_{t t} \cdots\right)=0$,

Where, $P$ is a polynomial in its arguments which includes nonlinear term and height order derivatives. Combining the independent variables $x$ and $t$ into one variable $\xi$, we suppose that

$u(x, t)=u(\xi), \xi=x-w t$,

Where $w \in \mathfrak{R}-\{0\}$ is the speed of the traveling wave, permits us to transform the Eq.(3) into an ordinary differential equation (ODE) :

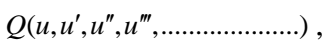

Where the superscripts stand for the ordinary derivatives with respect to $\xi$.

\subsection{The enhanced $\left(G^{\prime} / G\right)$-expansion method}

The main steps of enhanced $\left(G^{\prime} / G\right)^{-}$expansion method are to seek exact solutions of nonlinear partial differential equations can be given as follows [23]:

Step 1: Suppose the traveling wave solution of Eq. (5) can be expressed as follows:

$$
u(\xi)=\sum_{i=n}^{n}\left(\frac{a_{i}\left(G^{\prime} / G\right)^{i}}{\left(1+\lambda\left(G^{\prime} / G\right)\right)^{i}}+b_{i}\left(G^{\prime} / G\right)^{i-1} \sqrt{\sigma\left(1+\frac{\left(G^{\prime} / G\right)^{2}}{\mu}\right)}\right),
$$


Where the coefficients $a_{i}, b_{i}(-n \leq i \leq n, n \in \square)$ and $\lambda$ are constants to be determined later, and $\sigma= \pm 1, \mu \neq 0$. The positive integer $n$ can be determined by considering the homogeneous balance between the highest order derivatives and nonlinear terms appearing in Eq. (5). Precisely, we define the degree of $u(\xi)$ as $D(u(\xi))=n$, which gives rise to a degree of another expression as follows:

$D\left(\frac{d^{q} u}{d \xi^{q}}\right)=n+q, D\left(u^{p}\left(\frac{d^{q} u}{d \xi^{q}}\right)^{s}\right)=n p+s(n+q)$.

Therefore, we can insert the value of $n$ in Eq. (6) after finding the value of $n$ form Eq. (7).

Step-2: In this instance, $G=G(\xi)$ satisfies the following ordinary differential equation:

$G^{\prime \prime}(\xi)+\mu G(\xi)=0$,

It is notable that Eq. (8) has the following general solutions:

Type-I (Hyperbolic function solutions): When $\mu<0$

$\left(\frac{G^{\prime}}{G}\right)=\sqrt{-\mu} \tanh (A+\sqrt{-\mu} \xi)$, (9a)

$\left(\frac{G^{\prime}}{G}\right)=\sqrt{-\mu} \operatorname{coth}(A+\sqrt{-\mu} \xi)$,

Type-II (Trigonometric function solutions): When $\mu>0$

$\left(\frac{G^{\prime}}{G}\right)=\sqrt{\mu} \tan (A-\sqrt{\mu} \xi)$,

$\left(\frac{G^{\prime}}{G}\right)=\sqrt{\mu} \tan (A+\sqrt{\mu} \xi)$

Step 3: After we determine the index parameter $n$, we substitute Eq. (6) along with Eq.(8) into Eq.(5) and collecting all the terms of the same power of $\left(G^{\prime} / G\right)^{i}$ and $\left(G^{\prime} / G\right)^{i} \sqrt{\sigma\left(1+\frac{\left(G^{\prime} / G\right)^{2}}{\mu}\right)}$ together, equating them to zero, we obtain a system of algebraic equations, which can be solved by Maple to get the values of $a_{i}, b_{i}(-n \leq i \leq n, n \in \square), \lambda$ and $w$. Substituting the values of $a_{i}, b_{i}, \lambda$ and $w$ into Eq. (6) along with general solutions of Eq. (8) into Eq. (5), then we can derive exact solutions of Eq. (5).

\subsection{Improved $\left(1 / G^{\prime}\right)$ - expansion method}

The main steps of this method are to seek exact solutions of nonlinear partial differential equations can be given as follows (see details Khater and Kumar, [25]):

Step 1: Suppose the traveling wave solution of Eq. (5) can be expressed as follows:

$u(\xi)=\sum_{i=0}^{i=n} a_{i}(m+F(\xi))^{i}+\sum_{i=1}^{i=n} b_{i}(m+F(\xi))^{-i}$,

Where, $F(\xi)=1 / G^{\prime}(\xi)$ and the coefficients $a_{i}(0 \leq i \leq n, n \in \square)$ and $b_{i}(1 \leq i \leq n, n \in \square)$ are constants to be determined and either $a_{n}$ or $b_{n}$ may be zero but both $a_{n}$ and $b_{n}$ cannot be zero simultaneously. The positive integer $n$ can be determined by considering the homogeneous balance between the highest order derivatives and nonlinear terms appearing in Eq. (5). Therefore, we can find the value of $n$ from Eq. (7) in the same way of sub-section 2.1 .

Step-2: In this instance, $G=G(\xi)$ satisfies the following ordinary differential equation:

$G^{\prime \prime}(\xi)+\lambda G^{\prime}(\xi)+\mu=0$,

Where, $\lambda$ and $\mu$ are arbitrary constants.

Since $F=1 / G^{\prime}$, with the help of Eq. (12) we obtain, $F^{\prime}=\lambda F+\mu F^{2}$

The general solution of Eq. (12) is

$G(\xi)=-\frac{\mu \xi}{\lambda}-\frac{c_{1}}{\lambda} e^{-\lambda \xi}+c_{2}$

Where, $c_{1}$ and $c_{2}$ are two arbitrary constants. Then by differentiating Eq. (13) once with respect to $\xi$, we obtain the following expression: 
$F(\xi)=\frac{1}{G^{\prime}(\xi)}=\frac{1}{-\frac{\mu}{\lambda}+c_{1} e^{-\lambda \xi}}$

Equation (14) can be expressed by hyperbolic functions as follows:

$F(\xi)=\frac{1}{G^{\prime}(\xi)}=\frac{\lambda}{-\mu+\lambda A(\cosh (\lambda \xi)-\sinh (\lambda \xi))}, c_{1}=A$

Step 3: After we determine the index parameter $n$, we substitute Eq. (11) along with Eq. (12) into Eq. (5) and collecting all the terms of the same power $(F(\xi))^{i}, i=0,1,2, \ldots$. and equating them to zero, we obtain a system of algebraic equations, which can be solved by Maple

to get the values of $a_{i}, b_{i}, m, w$ and $V$, constant of integration. Substituting the values of $a_{i}, b_{i}, m, V$ and other values into Eq. (5) along with Eq. (12) completes the determination of the solution of Eq. (5).

\subsection{The $\left(G^{\prime} / G, 1 / G\right)$-expansion method}

In this sub-section, we describe the $\left(G^{\prime} / G, 1 / G\right)$-expansion method for finding traveling wave solutions of nonlinear evolution equations (NLEEs). Li. Et al. [26] has given $\left(G^{\prime} / G, 1 / G\right)$-expansion method as the preparations, consider the second-order linear ordinary differential equation (LODE):

$G^{\prime \prime}(\xi)+\lambda G(\xi)-\mu=0$

And we let

$\varphi=G^{\prime} / G, \psi=1 / G$

Using Eq. (16) and Eq. (17) yields

$\varphi^{\prime}=-\varphi^{2}+\mu \psi-\lambda, \psi^{\prime}=-\varphi \psi$

From the three cases of the general solutions of Eq. (16), we have:

Case 1: Hyperbolic function solutions

When $\lambda<0$, the general solution of Eq. (16) is,

$G(\xi)=A_{1} \sinh (\sqrt{-\lambda} \xi)+A_{2} \cosh (\sqrt{-\lambda} \xi)+\frac{\mu}{\lambda}$

And we have

$\psi^{2}=\frac{-\lambda}{\lambda^{2} \sigma+\mu^{2}}\left(\varphi^{2}-2 \mu \psi+\lambda\right)$

Where $A_{1}$ and $A_{2}$ are two arbitrary constants and $\sigma=A_{1}^{2}-A_{2}^{2}$.

Case 2: Trigonometric function solutions

When $\lambda>0$, the general solution of Eq. (16) is,

$G(\xi)=A_{1} \sin (\sqrt{\lambda} \xi)+A_{2} \cos (\sqrt{\lambda} \xi)+\frac{\mu}{\lambda}$

And we have

$\psi^{2}=\frac{\lambda}{\lambda^{2} \rho-\mu^{2}}\left(\varphi^{2}-2 \mu \psi+\lambda\right)$

Where $A_{1}$ and $A_{2}$ are two arbitrary constants and $\rho=A_{1}^{2}+A_{2}^{2}$.

Case 3: Rational function solutions

When $\lambda=0$, the general solution of Eq. (16) is,

$G(\xi)=\frac{\mu}{2} \xi^{2}+A_{1} \xi+A_{2}$

And we have 


$$
\psi^{2}=\frac{1}{A_{1}^{2}-2 \mu A_{2}}\left(\varphi^{2}-2 \mu \psi\right)
$$

Where $A_{1}$ and $A_{2}$ are two arbitrary constants.

In the following, we give the main steps of the $\left(G^{\prime} / G, 1 / G\right)$-expansion method, which is given in detail in reference [26], [27]:

Step 1: Suppose the traveling wave solution of Eq. (5) can be expressed by a polynomial in $\varphi$ and $\psi$ as follows:

$u(\xi)=\sum_{i=0}^{i=n} a_{i} \varphi^{i}+\sum_{i=1}^{i=n} b_{i} \varphi^{i-1} \psi$

Where $G=G(\xi)$ satisfies the Eq. (16), $a_{i}(i=0, \ldots, n), b_{i}(i=1, \ldots, n), V, \lambda$ and $\mu$ are constants to be determined later and the positive integer $n$ can be determined from Eq. (7), by using the same manner of sub-section 2.1.

Step 2: Substituting Eq. (25) into Eq. (5) and using Eq. (18) and Eq. (20) (here, Case -1 is taken as an example), the left-hand side of Eq. (5) can be converted into a polynomial in terms of $\varphi$ and $\psi$, in which the degree of $\psi$ is not larger than 1 and the degree of $\phi$ is from zero to an integer. Equating like power of the polynomial to zero yields a system of algebraic equations in $a_{i}(i=0, \ldots, n), b_{i}(i=1, \ldots, n)$, $w, \lambda(\lambda<0), \mu, A_{1}$ and $A_{2}$.

Step 3: Solving the algebraic equation attained in Step 2 with the aid of Maple. We get the value of, $b_{i}, w, \lambda(\lambda<0), \mu, A_{1}$ and $A_{2}$. Substituting the values $a_{i}(i=0, \ldots, n), b_{i}(i=1, \ldots, n), w, \lambda(\lambda<0), \mu, A_{1}$ and $A_{2}$ obtained into Eq. (25), one can obtain the travelling wave solutions expressed by hyperbolic functions of Eq. (5).

Step 4: Similar to Steps 3 and 4, substituting Eq. (25) into Eq. (5) and using Eq. (18) and Eq. (22) (or (18) and (24)), we obtain the travelling wave solutions of Eq. (5) expressed by trigonometric functions (or expressed by rational functions).

\section{Applications}

In this section, we will implement the mentioned methods described in section 2, to find the new exact solution of the Tzitzeica-DoddBullough (TDB) equation. Using the Painleave transformation, $v=e^{-u}$ or $u=-\ln (v)$, Eq. (2) transforms into the following partial differential equation,

$$
v v_{x t}-v_{x} v_{t}+v^{3}+v^{4}=0
$$

Utilizing the assume transformation of Eq. (4), then Eq. (26) convert into the following ordinary differential equation:

$-w v v^{\prime \prime}+w\left(v^{\prime}\right)^{2}+v^{3}+v^{4}=0$

Where prime denotes the derivation with respect to $\xi$.

\subsection{Application of the enhanced $\left(G^{\prime} / G\right)$-expansion method}

In this sub-section, the enhanced $\left(G^{\prime} / G\right)$-expansion method described in sub-section 2.1 , has been implemented to derive exact solutions of the Tzitzeica-Dodd-Bullough (TDB) equation. Now, balancing the order of $v v^{\prime \prime}$ and $v^{4}$ from Eq. (27), yields $2 n+2=4 n$ and we get $n=1$. So, we consider the following formal solution of Eq. (27):

$v(\xi)=\left(\begin{array}{l}\frac{a_{-1}\left(1+\lambda\left(G^{\prime} / G\right)\right)}{\left(G^{\prime} / G\right)}+\frac{b_{-1} \sqrt{\sigma\left(1+\frac{\left(G^{\prime} / G\right)^{2}}{\mu}\right)}}{\left(G^{\prime} / G\right)^{2}}+a_{0}+\frac{b_{0} \sqrt{\sigma\left(1+\frac{\left(G^{\prime} / G\right)^{2}}{\mu}\right)}}{\left(G^{\prime} / G\right)} \\ +\frac{a_{1}\left(G^{\prime} / G\right)}{1+\lambda\left(G^{\prime} / G\right)}+b_{1} \sqrt{\sigma\left(1+\frac{\left(G^{\prime} / G\right)^{2}}{\mu}\right)}\end{array}\right)$

Where the coefficients $a_{-1}, a_{0}, \alpha_{1}, b_{-1}, b_{0}$ and $b_{1}$ are arbitrary constants to be determined later. Substituting Eq. (28) into Eq. (27) along with Eq.(8), the left hand side of Eq. (27) becomes a polynomial in $\left(G^{\prime} / G\right)$ and $\left(G^{\prime} / G\right) \sqrt{\sigma\left(1+\frac{\left(G^{\prime} / G\right)^{2}}{\mu}\right)}$, setting each coefficient of this polynomial to zero, yields a system of algebraic equations (for simplicity, we omitted to display them) in terms of $a_{-1}, a_{0}, \alpha_{1}, b_{-1}, b_{0}, b_{1}, \mu$ and $\lambda$, Solving these resulting system using the symbolic computation package, gives: 
Set $1:\left\{w=-\frac{1}{4 \mu}, a_{-1}=0, a_{0}=-\frac{1}{2} \pm \frac{i}{2} \lambda \sqrt{\mu}, a_{1}= \pm \frac{1}{2} \frac{\mu \lambda^{2}+1}{i \sqrt{\mu}}, b_{-1}=0, b_{0}=0, b_{1}=0\right\}$

Set 2: $\left\{w=\frac{1}{4 \lambda^{2} \mu^{2}}, a_{-1}=\frac{1}{2 \lambda}, a_{0}=-1, a_{1}=\frac{1}{2} \frac{\mu \lambda^{2}+1}{\lambda \mu}, b_{-1}=0, b_{0}=0, b_{1}=0\right\}$

Set 3: $\left\{w=-\frac{1}{4 \mu}, a_{-1}= \pm \frac{i}{2} \sqrt{\mu}, a_{0}=-\frac{1}{2} \pm \frac{i}{2} \lambda \sqrt{\mu}, a_{1}=0, b_{-1}=0, b_{0}=0, b_{1}=0\right\}$

Set 4: $\left\{w=-\frac{1}{\mu}, a_{-1}=\frac{i}{2} \sqrt{\mu}, a_{0}=-\frac{1}{2}-\frac{i}{2} \lambda \sqrt{\mu}, a_{1}=0, b_{-1}=0, b_{0}= \pm \frac{i}{2} \frac{\sqrt{\mu \sigma}}{\sigma}, b_{1}=0\right\}$

Set 5: $\left\{w=-\frac{1}{\mu}, a_{-1}=-\frac{i}{2} \sqrt{\mu}, a_{0}=-\frac{1}{2}+\frac{i}{2} \lambda \sqrt{\mu}, a_{1}=0, b_{-1}=0, b_{0}= \pm \frac{i}{2} \frac{\sqrt{\mu \sigma}}{\sigma}, b_{1}=0\right\}$

Now substituting set- 1 to set-5 with the general solutions of Eq. (8) into Eq. (28), and using the transformation $u=-\ln v$, we obtain abundant travelling wave solutions of Eq. (2) as follows:

Case-1 (Hyperbolic function solutions): When $\mu<0$, we get the following hyperbolic function solutions:

$$
\begin{aligned}
& u_{1,2}(x, t)=-\ln \left(-\frac{1}{2} \pm \frac{i}{2} \lambda \sqrt{\mu} \pm \frac{\left(\mu \lambda^{2}+1\right) \tanh \left(A+i \sqrt{\mu}\left(x-\frac{t}{4 \mu}\right)\right)}{2+2 i \lambda \sqrt{\mu} \tanh \left(A+i \sqrt{\mu}\left(x-\frac{t}{4 \mu}\right)\right)}\right) \\
& u_{3,4}(x, t)=-\ln \left(-\frac{1}{2} \pm \frac{i}{2} \lambda \sqrt{\mu} \pm \frac{\left(\mu \lambda^{2}+1\right) \operatorname{coth}\left(A+i \sqrt{\mu}\left(x-\frac{t}{4 \mu}\right)\right)}{2+2 i \lambda \sqrt{\mu} \operatorname{coth}\left(A+i \sqrt{\mu}\left(x-\frac{t}{4 \mu}\right)\right)}\right) \\
& u_{5}(x, t)=-\ln \left(\begin{array}{l}
\frac{1+i \lambda \sqrt{\mu} \tanh \left(A+i \sqrt{\mu}\left(x+\frac{t}{4 \lambda^{2} \mu^{2}}\right)\right)}{2 i \lambda \sqrt{\mu} \tanh \left(A+i \sqrt{\mu}\left(x+\frac{t}{4 \lambda^{2} \mu^{2}}\right)\right)}-1 \\
\left.+\frac{\left(\mu \lambda^{2}+1\right) i \sqrt{\mu} \tanh \left(A+i \sqrt{\mu}\left(x+\frac{t}{4 \lambda^{2} \mu^{2}}\right)\right)}{2 \lambda \mu+2 i \mu \lambda^{2} \sqrt{\mu} \tanh \left(A+i \sqrt{\mu}\left(x+\frac{t}{4 \lambda^{2} \mu^{2}}\right)\right)}\right)
\end{array}\right) \\
& u_{6}(x, t)=-\ln \left(\begin{array}{l}
\frac{1+i \lambda \sqrt{\mu} \operatorname{coth}\left(A+\sqrt{\mu}\left(x+\frac{t}{4 \lambda^{2} \mu^{2}}\right)\right)}{2 i \lambda \sqrt{\mu} \operatorname{coth}\left(A+\sqrt{\mu}\left(x+\frac{t}{4 \lambda^{2} \mu^{2}}\right)\right)}-1 \\
\left.+\frac{\left(\mu \lambda^{2}+1\right) i \sqrt{\mu} \operatorname{coth}\left(A+i \sqrt{\mu}\left(x+\frac{t}{4 \lambda^{2} \mu^{2}}\right)\right)}{2 \mu \lambda+2 i \mu \lambda^{2} \sqrt{\mu} \operatorname{coth}\left(A+i \sqrt{\mu}\left(x+\frac{t}{4 \lambda^{2} \mu^{2}}\right)\right)}\right)
\end{array}\right) \\
& u_{7,8}(x, t)=-\ln \left( \pm \frac{1+i \lambda \sqrt{\mu} \tanh \left(A+i \sqrt{\mu}\left(x-\frac{t}{4 \mu}\right)\right)}{2 \tanh \left(A+i \sqrt{\mu}\left(x-\frac{t}{4 \mu}\right)\right)}-\frac{1}{2} \pm \frac{i}{2} \lambda \sqrt{\mu}\right)
\end{aligned}
$$


$u_{9,10}(x, t)=-\ln \left( \pm \frac{1+i \lambda \sqrt{\mu} \operatorname{coth}\left(A+i \sqrt{\mu}\left(x-\frac{t}{4 \mu}\right)\right)}{2 \operatorname{coth}\left(A+i \sqrt{\mu}\left(x-\frac{t}{4 \mu}\right)\right)}-\frac{1}{2} \pm \frac{i}{2} \lambda \sqrt{\mu}\right)$

$$
u_{11,1,2}(x, t)=-\ln \left(\begin{array}{l}
\frac{1+i \lambda \sqrt{\mu} \tanh \left(A+i \sqrt{\mu}\left(x-\frac{t}{\mu}\right)\right)}{2 \tanh \left(A+i \sqrt{\mu}\left(x-\frac{t}{\mu}\right)\right)}-\frac{1}{2}-\frac{i}{2} \lambda \sqrt{\mu} \\
\pm \frac{i \sqrt{\mu \sigma} \sqrt{\sigma\left(1-\tanh \left(A+i \sqrt{\mu}\left(x-\frac{t}{\mu}\right)\right)^{2}\right)}}{2 \sigma i \sqrt{\mu} \tanh \left(A+i \sqrt{\mu}\left(x-\frac{t}{\mu}\right)\right)}
\end{array}\right)
$$

$u_{13,14}(x, t)=-\ln \left(\begin{array}{l}\frac{1+i \lambda \sqrt{\mu} \operatorname{coth}\left(A+i \sqrt{\mu}\left(x-\frac{t}{\mu}\right)\right)}{2 \operatorname{coth}\left(A+i \sqrt{\mu}\left(x-\frac{t}{\mu}\right)\right)}-\frac{1}{2}-\frac{i}{2} \lambda \sqrt{\mu} \\ \pm \frac{i \sqrt{\mu \sigma} \sqrt{\left(1-\operatorname{coth}\left(A+i \sqrt{\mu}\left(x-\frac{t}{\mu}\right)\right)^{2}\right)}}{2 i \sigma \sqrt{\mu} \operatorname{coth}\left(A+i \sqrt{\mu}\left(x-\frac{t}{\mu}\right)\right)}\end{array}\right)$

$u_{15,16}(x, t)=-\ln \left(\begin{array}{l}-\frac{1+i \lambda \sqrt{\mu} \tanh \left(A+i \sqrt{\mu}\left(x-\frac{t}{\mu}\right)\right)}{2 \tanh \left(A+i \sqrt{\mu}\left(x-\frac{t}{\mu}\right)\right)}-\frac{1}{2}+\frac{i}{2} \lambda \sqrt{\mu} \\ \pm \frac{i \sqrt{\mu \sigma} \sqrt{\sigma\left(1-\tanh \left(A+i \sqrt{\mu}\left(x-\frac{t}{\mu}\right)\right)^{2}\right)}}{2 i \sigma \sqrt{\mu} \tanh \left(A+i \sqrt{\mu}\left(x-\frac{t}{\mu}\right)\right)}\end{array}\right)$

$$
u_{17,18}(x, t)=-\ln \left(\begin{array}{l}
-\frac{1+i \lambda \sqrt{\mu} \operatorname{coth}\left(A+i \sqrt{\mu}\left(x-\frac{t}{\mu}\right)\right)}{2 \operatorname{coth}\left(A+i \sqrt{\mu}\left(x-\frac{t}{\mu}\right)\right)}-\frac{1}{2}+\frac{i}{2} \lambda \sqrt{\mu} \\
\pm \frac{i \sqrt{\mu \sigma} \sqrt{\left(1-\operatorname{coth}\left(A+i \sqrt{\mu}\left(x-\frac{t}{\mu}\right)\right)^{2}\right)}}{2 i \sigma \sqrt{\mu} \operatorname{coth}\left(A+i \sqrt{\mu}\left(x-\frac{t}{\mu}\right)\right)}
\end{array}\right)
$$

Case-2 (Trigonometric function solutions): When $\mu>0$, we derive the following trigonometric function solutions:

$$
\begin{aligned}
& u_{19,20}(x, t)=-\ln \left(-\frac{1}{2} \pm \frac{i}{2} \lambda \sqrt{\mu} \pm \frac{\left(\mu \lambda^{2}+1\right) \sqrt{\mu} \tan \left(A-\sqrt{\mu}\left(x-\frac{t}{4 \mu}\right)\right)}{2 i \sqrt{\mu}-2 \mu \lambda \tan \left(A-\sqrt{\mu}\left(x-\frac{t}{4 \mu}\right)\right)}\right) \\
& u_{21,22}(x, t)=-\ln \left(-\frac{1}{2} \pm \frac{i}{2} \lambda \sqrt{\mu} \pm \frac{\left(\mu \lambda^{2}+1\right) \sqrt{\mu} \cot \left(A+\sqrt{\mu}\left(x-\frac{t}{4 \mu}\right)\right)}{2 i \sqrt{\mu}-2 \mu \lambda \cot \left(A+\sqrt{\mu}\left(x-\frac{t}{4 \mu}\right)\right)}\right)
\end{aligned}
$$


$u_{23}(x, t)=-\ln \left(\begin{array}{l}\frac{1+\lambda \sqrt{\mu} \tan \left(A-\sqrt{\mu}\left(x+\frac{t}{4 \lambda^{2} \mu^{2}}\right)\right)}{2 \sqrt{\mu} \tan \left(A-\sqrt{\mu}\left(x+\frac{t}{4 \lambda^{2} \mu^{2}}\right)\right) \lambda}-1 \\ \left.+\frac{\left(\mu \lambda^{2}+1\right) \tan \left(A-\sqrt{\mu}\left(x+\frac{t}{4 \lambda^{2} \mu^{2}}\right)\right)}{2 \sqrt{\mu}+2 \lambda^{2} \mu \tan \left(A-\sqrt{\mu}\left(x+\frac{t}{4 \lambda^{2} \mu^{2}}\right)\right)}\right)\end{array}\right)$

$u_{24}(x, t)=-\ln \left(\begin{array}{l}\frac{1+\lambda \sqrt{\mu} \cot \left(A+\sqrt{\mu}\left(x+\frac{t}{4 \lambda^{2} \mu^{2}}\right)\right)}{2 \sqrt{\mu} \cot \left(A+\sqrt{\mu}\left(x+\frac{t}{4 \lambda^{2} \mu^{2}}\right)\right) \lambda}-1 \\ \left.+\frac{\left(\mu \lambda^{2}+1\right) \cot \left(A+\sqrt{\mu}\left(x+\frac{t}{4 \lambda^{2} \mu^{2}}\right)\right)}{2 \sqrt{\mu}+2 \mu \lambda^{2} \cot \left(A+\sqrt{\mu}\left(x+\frac{t}{4 \lambda^{2} \mu^{2}}\right)\right)}\right)\end{array}\right)$

$u_{25,26}(x, t)=-\ln \left( \pm \frac{i \sqrt{\mu}-\mu \lambda \tan \left(A-i \sqrt{\mu}\left(x-\frac{t}{4 \mu}\right)\right)}{2 \sqrt{\mu} \tan \left(A-i \sqrt{\mu}\left(x-\frac{t}{4 \mu}\right)\right)}-\frac{1}{2} \pm \frac{i}{2} \lambda \sqrt{\mu}\right)$

$u_{27,28}(x, t)=-\ln \left( \pm \frac{i \sqrt{\mu}-\mu \lambda \cot \left(A+\sqrt{\mu}\left(x-\frac{t}{4 \mu}\right)\right)}{2 \sqrt{\mu} \cot \left(A+\sqrt{\mu}\left(x-\frac{t}{4 \mu}\right)\right)}-\frac{1}{2} \pm \frac{i}{2} \lambda \sqrt{\mu}\right)$

$u_{29,30}(x, t)=-\ln \left(\begin{array}{l}\frac{i \sqrt{\mu}-\mu \lambda \tan \left(A-\sqrt{\mu}\left(x-\frac{t}{\mu}\right)\right)}{2 \sqrt{\mu} \tan \left(A-\sqrt{\mu}\left(x-\frac{t}{\mu}\right)\right)}-\frac{1}{2} \\ -\frac{i \lambda \sqrt{\mu}}{2} \pm \frac{-\sqrt{\left(1+\tan \left(A-\sqrt{\mu}\left(x-\frac{t}{\mu}\right)\right)^{2}\right)}}{2 \tan \left(A-\sqrt{\mu}\left(x-\frac{t}{\mu}\right)\right)}\end{array}\right)$

$u_{3,1,2}(x, t)=-\ln \left(\begin{array}{l}\frac{i \sqrt{\mu}-\mu \lambda \cot \left(A-\sqrt{\mu}\left(x-\frac{t}{\mu}\right)\right)}{2 \sqrt{\mu} \cot \left(A-\sqrt{\mu}\left(x-\frac{t}{\mu}\right)\right)}-\frac{1}{2} \\ -\frac{i \lambda \sqrt{\mu}}{2} \pm \frac{-\sqrt{\left(1+\cot \left(A-\sqrt{\mu}\left(x-\frac{t}{\mu}\right)\right)^{2}\right)}}{2 \cot \left(A-\sqrt{\mu}\left(x-\frac{t}{\mu}\right)\right)}\end{array}\right)$

$u_{33,34}(x, t)=-\ln \left(\begin{array}{l}-\frac{i \sqrt{\mu}-\mu \lambda \tan \left(A-\sqrt{\mu}\left(x-\frac{t}{\mu}\right)\right)}{2 \sqrt{\mu} \tan \left(A-\sqrt{\mu}\left(x-\frac{t}{\mu}\right)\right)}-\frac{1}{2}+\frac{i \lambda \sqrt{\mu}}{2} \\ \pm \frac{-\sqrt{\left(1+\tan \left(A-\sqrt{\mu}\left(x-\frac{t}{\mu}\right)\right)^{2}\right)}}{2 \tan \left(A-\sqrt{\mu}\left(x-\frac{t}{\mu}\right)\right)}\end{array}\right)$ 


$$
u_{35,36}(x, t)=-\ln \left(\begin{array}{l}
-\frac{i \sqrt{\mu}-\mu \lambda \cot \left(A+\sqrt{\mu}\left(x-\frac{t}{\mu}\right)\right)}{2 \sqrt{\mu} \cot \left(A+\sqrt{\mu}\left(x-\frac{t}{\mu}\right)\right)}-\frac{1}{2}+\frac{i \lambda \sqrt{\mu}}{2} \\
\pm \frac{-\sqrt{\left(1+\cot \left(A+\sqrt{\mu}\left(x-\frac{t}{\mu}\right)\right)^{2}\right)}}{2 \cot \left(A+\sqrt{\mu}\left(x-\frac{t}{\mu}\right)\right)}
\end{array}\right)
$$

\subsection{Application of the improved ${ }^{\left(1 / G^{\prime}\right)}$-expansion method}

In this section, the improved $\left(1 / G^{\prime}\right)$-expansion method delineated in the sub-section 2.2 has been implemented to derive new exact solutions of the Tzitzeica-Dodd-Bullough (TDB) equation. Assume the formal solution of Eq. (27) is the following form:

$v(\xi)=a_{0}+a_{1}(m+F(\xi))+b_{1}(m+F(\xi))^{-1}$

Where the coefficients $a_{0} a_{1}, \mathrm{~b}_{1}$ and $m$ are arbitrary constants to be determined later. Substituting Eq. (50) into Eq. (27) along with Eq. (12), the left-hand side of Eq. (27) becomes a polynomial in $F(\xi)$, setting each coefficient of this polynomial to zero yield a system of algebraic equations of $a_{0}, a_{1}, b_{1}, \mu, \lambda, m$ and $w$ (for simplicity we omitted to display them). Solving these resulting systems using the symbolic computation package, gives:

Set 1: $\left\{m=m, w=\frac{1}{\lambda^{2}}, \alpha_{0}=\frac{m \mu-\lambda}{\lambda}, \alpha_{1}=0, \beta_{1}=-\frac{m(m \mu-\lambda)}{\lambda}\right\}$

Set 2: $\left\{m=-\frac{\alpha_{0} \lambda}{\mu}, w=\frac{1}{\lambda^{2}}, \alpha_{0}=\alpha_{0}, \alpha_{1}=0, \beta_{1}=\frac{\alpha_{0} \lambda\left(\alpha_{0}+1\right)}{\mu}\right\}$

Set 3: $\left\{m=m, w=\frac{1}{\lambda^{2}}, \alpha_{0}=\frac{m \mu-\lambda}{\lambda}, \alpha_{1}=-\frac{\mu}{\lambda}, \beta_{1}=0\right\}$

Set 4: $\left\{m=m, w=\frac{1}{\lambda^{2}}, \alpha_{0}=-\frac{m \mu}{\lambda}, \alpha_{1}=\frac{\mu}{\lambda}, \beta_{1}=0\right\}$

Set 5: $\left\{m=\frac{1}{2} \frac{\lambda \alpha_{1}+\mu}{\mu \alpha_{1}}, w=\frac{\alpha_{1}^{2}}{\mu^{2}}, \alpha_{0}=-1, \alpha_{1}=\alpha_{1}, \beta_{1}=\frac{1}{4} \frac{-\lambda^{2} \alpha_{1}^{2}+\mu^{2}}{\mu^{2} \alpha_{1}}\right\}$

Set 6: $\left\{m=\frac{1}{2} \frac{\lambda}{\mu}, w=\frac{1}{4 \lambda^{2}}, \alpha_{0}=-\frac{1}{2}, \alpha_{1}= \pm \frac{1}{2} \frac{\mu}{\lambda}, \beta_{1}= \pm \frac{1}{8} \frac{\lambda}{\mu}\right\}$

Now substituting set-1 to set-6 with Eq. (15) into Eq. (50), and using the transformation $u=-\ln v$, we constructed abundant travelling wave solutions of Eq. (2) as follows:

$$
\begin{aligned}
& u_{1}(x, t)=-\ln \left(\frac{m \mu-\lambda}{\lambda}-\frac{m(m \mu-\lambda)\left(-\mu+A \lambda \cosh \left(\lambda x-\frac{t}{\lambda}\right)-A \lambda \sinh \left(\lambda x-\frac{t}{\lambda}\right)\right)}{\left.-m \lambda \mu+A \lambda^{2} m\left(\cosh \left(\lambda x-\frac{t}{\lambda}\right)-\sinh \left(\lambda x-\frac{t}{\lambda}\right)\right)+\lambda^{2}\right)}\right) \\
& u_{2}(x, t)=-\ln \left(\begin{array}{c}
\left.\alpha_{0}+\frac{\lambda \alpha_{0}\left(\alpha_{0}+1\right)\left(-\mu+\lambda A \cosh \left(\lambda x-\frac{t}{\lambda}\right)-\lambda A \sinh \left(\lambda x-\frac{t}{\lambda}\right)\right)}{\alpha_{0} \lambda \mu-\alpha_{0} \lambda^{2} A\left(\cosh \left(\lambda x-\frac{t}{\lambda}\right)-\sinh \left(\lambda x-\frac{t}{\lambda}\right)\right)+\lambda \mu}\right) \\
u_{3}(x, t)=-\ln \left(\frac{m \mu-\lambda}{\lambda}-\frac{-m \mu^{2}+m A \lambda \mu\left(\cosh \left(\lambda x-\frac{t}{\lambda}\right)-\sinh \left(\lambda x-\frac{t}{\lambda}\right)\right)+\lambda \mu}{\lambda-\mu+\lambda A\left(\cosh \left(\lambda x-\frac{t}{\lambda}\right)-\sinh \left(\lambda x-\frac{t}{\lambda}\right)\right)}\right)
\end{array}\right)
\end{aligned}
$$




$$
\begin{aligned}
& u_{4}(x, t)=-\ln \left(-\frac{m \mu}{\lambda}+\frac{-m \mu^{2}+\lambda A\left(\cosh \left(\lambda x-\frac{t}{\lambda}\right)-\sinh \left(\lambda x-\frac{t}{\lambda}\right)\right)+\lambda \mu}{\lambda}\right) \\
& u_{5}(x, t)=-\ln \left(\begin{array}{c}
-1+\frac{\left(\lambda \alpha_{1}+\mu\right)\left(-\mu+\lambda A \cosh \left(\lambda x-\frac{\alpha_{1}^{2} \lambda t}{\mu^{2}}\right)-\lambda A \sinh \left(\lambda x-\frac{\alpha_{1}^{2} \lambda t}{\mu^{2}}\right)\right)+2 \mu \alpha_{1} \lambda}{2 \mu\left(-\mu+\lambda A \cosh \left(\lambda x-\frac{\alpha_{1}^{2} \lambda t}{\mu^{2}}\right)-\lambda A \sinh \left(\lambda x-\frac{\alpha_{1}^{2} \lambda t}{\mu^{2}}\right)\right)} \\
+\frac{1}{2 \mu} \frac{\left(-\lambda^{2} \alpha_{1}^{2}+\mu^{2}\right)\left(-\mu+\lambda A \cosh \left(\lambda x-\frac{\alpha_{1}^{2} \lambda t}{\mu^{2}}\right)-\lambda A \sinh \left(\lambda x-\frac{\alpha_{1}^{2} \lambda t}{\mu^{2}}\right)\right)}{\left(\lambda \alpha_{1}+\mu\right)\left(-\mu+\lambda A \cosh \left(\lambda x-\frac{\alpha_{1}^{2} \lambda t}{\mu^{2}}\right)-\lambda A \sinh \left(\lambda x-\frac{\alpha_{1}^{2} \lambda t}{\mu^{2}}\right)\right)+2 \lambda \mu \alpha_{1}}
\end{array}\right) \\
& u_{6,7}(x, t)=-\ln \left(\begin{array}{l}
-\frac{1}{2} \pm \frac{1}{2} \frac{\lambda^{2}\left(-\mu+\lambda A \cosh \left(\lambda x-\frac{t}{4 \lambda}\right)-\lambda A \sinh \left(\lambda x-\frac{t}{4 \lambda}\right)\right)+2 \mu \lambda^{2}}{2\left(-\mu+\lambda A \cosh \left(\lambda x-\frac{t}{4 \lambda}\right)-\lambda A \sinh \left(\lambda x-\frac{t}{4 \lambda}\right)\right)} \\
\pm \frac{1}{4} \frac{\left(-\mu+\lambda A \cosh \left(\lambda x-\frac{t}{4 \lambda}\right)-\lambda A \sinh \left(\lambda x-\frac{t}{4 \lambda}\right)\right)}{\left(-\mu+\lambda A \cosh \left(\lambda x-\frac{t}{4 \lambda}\right)-\lambda A \sinh \left(\lambda x-\frac{t}{4 \lambda}\right)\right)+2 \mu \lambda}
\end{array}\right)
\end{aligned}
$$

\subsection{Application of the $\left(G^{\prime} / G, 1 / G\right)$-expansion method}

In this section, the two variables $\left(G^{\prime} / G, 1 / G^{\prime}\right)$ - expansion method impressed in sub-section 2.3 has been implemented to explore the new exact solutions of the Tzitzeica-Dodd-Bullough (TDB) equation. Therefore, the formal considered solution of Eq. (27) is the following form:

$$
v(\xi)=a_{0}+a_{1} \phi(\xi)+b_{1} \psi(\xi)
$$

Where the coefficients $a_{0} a_{1}$ and $b_{1}$ are arbitrary constants to be determined later. There are three cases to discuss the solutions of Eq. (27), as given in section 2.3 .

Case 1: When $\lambda<0$ (Hyperbolic function solutions)

Substituting Eq. (57) into Eq. (27) with the help of Eq. (18) and Eq. (20), the left-hand side of Eq. (27) becomes a polynomial in $\phi$ and $\psi$, setting each coefficient of this polynomial to zero yield a system of algebraic equations in $a_{0}, a_{1}, \mu, \lambda$ and $w$. Solving these resulting systems using the symbolic computation package, gives:

Set-1: $\left\{w=-\frac{1}{\lambda}, a_{0}=-\frac{1}{2}, a_{1}=\frac{i}{2 \sqrt{\lambda}}, b_{1}= \pm \frac{1}{2} \frac{\sqrt{\mu^{2}+\lambda^{2}\left(A_{1}^{2}-A_{2}^{2}\right)}}{\lambda}\right\}$

Set- $2:\left\{w=-\frac{1}{\lambda}, a_{0}=-\frac{1}{2}, a_{1}=-\frac{i}{2 \sqrt{\lambda}}, b_{1}= \pm \frac{1}{2} \frac{\sqrt{\mu^{2}+\lambda^{2}\left(A_{1}^{2}-A_{2}^{2}\right)}}{\lambda}\right\}$

Set-3: $\left\{w=-\frac{\mu^{2}+\lambda^{2}\left(A_{1}^{2}-A_{2}^{2}\right)}{\lambda \mu^{2}}, a_{0}=0, a_{1}=0, b_{1}=-\frac{\mu^{2}+\lambda^{2}\left(A_{1}^{2}-A_{2}^{2}\right)}{\lambda \mu}\right\}$

Now substituting set-1 to set-3 into Eq. (57) and using the transformation $u=-\ln (v)$, we get 


$$
\begin{aligned}
& u_{1,2}(x, t)=-\ln \left(\begin{array}{l}
-\frac{1}{2}+\frac{\frac{1}{2}\left(A_{1} \cosh \left(i x \sqrt{\lambda}+\frac{i}{\sqrt{\lambda}} t\right)+A_{2} \sinh \left(i x \sqrt{\lambda}+\frac{i}{\sqrt{\lambda}} t\right)\right)}{A_{1} \sinh \left(i x \sqrt{\lambda}+\frac{i}{\sqrt{\lambda}} t\right)+A_{2} \cosh \left(i x \sqrt{\lambda}+\frac{i}{\sqrt{\lambda}} t\right)+\frac{\mu}{\lambda}} \\
\pm \frac{1}{2 \lambda} \frac{\sqrt{\mu^{2}+\lambda^{2}\left(A_{1}^{2}-A_{2}^{2}\right)}}{A_{1} \sinh \left(i x \sqrt{\lambda}+\frac{i}{\sqrt{\lambda}} t\right)+A_{2} \cosh \left(i x \sqrt{\lambda}+\frac{i}{\sqrt{\lambda}} t\right)+\frac{\mu}{\lambda}}
\end{array}\right) \\
& u_{3,4}(x, t)=-\ln \left(\begin{array}{l}
-\frac{1}{2}-\frac{\frac{1}{2}\left(A_{1} \cosh \left(i x \sqrt{\lambda}+\frac{i}{\sqrt{\lambda}} t\right)+A_{2} \sinh \left(i x \sqrt{\lambda}+\frac{i}{\sqrt{\lambda}} t\right)\right)}{A_{1} \sinh \left(i x \sqrt{\lambda}+\frac{i}{\sqrt{\lambda}} t\right)+A_{2} \cosh \left(i x \sqrt{\lambda}+\frac{i}{\sqrt{\lambda}} t\right)+\frac{\mu}{\lambda}} \\
\pm \frac{1}{2 \lambda} \frac{\sqrt{\mu^{2}+\lambda^{2}\left(A_{1}^{2}-A_{2}^{2}\right)}}{A_{1} \sinh \left(i x \sqrt{\lambda}+\frac{i}{\sqrt{\lambda}} t\right)+A_{2} \cosh \left(i x \sqrt{\lambda}+\frac{i}{\sqrt{\lambda}} t\right)+\frac{\mu}{\lambda}}
\end{array}\right) \\
& u_{5}(x, t)=-\ln \left(-\frac{\mu^{2}+\lambda^{2}\left(A_{1}^{2}-A_{2}^{2}\right)}{\mu \lambda\left(A_{1} \sinh \left(\frac{i \lambda \mu^{2} x-i\left(\mu^{2}+\lambda^{2} \sigma\right) t}{\mu^{2} \sqrt{\lambda}}\right)+A_{2} \cosh \left(\frac{i \lambda \mu^{2} x-i\left(\mu^{2}+\lambda^{2} \sigma\right) t}{\mu^{2} \sqrt{\lambda}}\right)+\mu^{2}\right.}\right)
\end{aligned}
$$

Solution Eq. (58) to Eq. (60) are the generalized closed form solutions of the Tzitzeica-Dodd-Bullough equation, where $A_{1}$ and $A_{2}$ are arbitrary constants. In particular, if we set $A_{1}=0, A_{2} \neq 0$ and $\mu=0$ then from Eq. (58) to Eq. (59), we obtain a new type of solitary wave solutions, given in the following:

$$
\begin{aligned}
& u_{6,7}(x, t)=-\ln \left(-\frac{1}{2}+\frac{1}{2} \tanh \left(i x \sqrt{\lambda}+\frac{i}{\sqrt{\lambda}} t\right) \pm \frac{1}{2} \operatorname{sech}\left(i x \sqrt{\lambda}+\frac{i}{\sqrt{\lambda}} t\right)\right) \\
& u_{8,9}(x, t)=-\ln \left(-\frac{1}{2}-\frac{1}{2} \tanh \left(i x \sqrt{\lambda}+\frac{i}{\sqrt{\lambda}} t\right) \pm \frac{1}{2} \operatorname{sech}\left(i x \sqrt{\lambda}+\frac{i}{\sqrt{\lambda}} t\right)\right)
\end{aligned}
$$

On the other hand, for $A_{1} \neq 0, A_{2}=0$ and $\mu=0$, from Eq. (58) - (59) we obtained the following solitary wave solutions respectively:

$$
\begin{aligned}
& u_{10,11}(x, t)=-\ln \left(-\frac{1}{2}+\frac{1}{2} \operatorname{coth}\left(i x \sqrt{\lambda}+\frac{i}{\sqrt{\lambda}} t\right) \pm \frac{1}{2} \operatorname{csch}\left(i x \sqrt{\lambda}+\frac{i}{\sqrt{\lambda}} t\right)\right) \\
& u_{12,13}(x, t)=-\ln \left(-\frac{1}{2}-\frac{1}{2} \operatorname{coth}\left(i x \sqrt{\lambda}+\frac{i}{\sqrt{\lambda}} t\right) \pm \frac{1}{2} \operatorname{csch}\left(i x \sqrt{\lambda}+\frac{i}{\sqrt{\lambda}} t\right)\right)
\end{aligned}
$$

Case 2: When $\lambda>0$ (Trigonometric function solutions)

Taking the same steps as for case- 1 and solving the system of equations yields sets of values of arbitrary constants as follows:

Set 4: $\left\{w=-\frac{1}{\lambda}, a_{0}=-\frac{1}{2}, a_{1}=\sqrt{-\frac{1}{4 \lambda}}, b_{1}= \pm \frac{1}{2} \frac{\sqrt{\mu^{2}-\lambda^{2}\left(A_{1}^{2}+A_{2}^{2}\right)}}{\lambda}\right\}$

Set 5: $\left\{w=-\frac{1}{\lambda}, a_{0}=-\frac{1}{2}, a_{1}=-\sqrt{-\frac{1}{4 \lambda}}, b_{1}= \pm \frac{1}{2} \frac{\sqrt{\mu^{2}-\lambda^{2}\left(A_{1}^{2}+A_{2}^{2}\right)}}{\lambda}\right\}$

Set 6: $\left\{w=-\frac{\mu^{2}-\lambda^{2}\left(A_{1}^{2}+A_{2}^{2}\right)}{\lambda \mu^{2}}, a_{0}=0, a_{1}=0, b_{1}=-\frac{\mu^{2}-\lambda^{2}\left(A_{1}^{2}+A_{2}^{2}\right)}{\lambda \mu}\right\}$

Now substituting set- 4 to set- 6 into Eq. (57) and using the transformation $u=-\ln (v)$, the following general solitary wave solutions of Eq. (2) are obtained: 


$$
\begin{aligned}
& u_{14,15}(x, t)=-\ln \left(\begin{array}{l}
-\frac{1}{2}+\frac{-\frac{1}{2}\left(A_{1} \cos \left(x \sqrt{\lambda}+\frac{1}{\sqrt{\lambda}} t\right)+A_{2} \sin \left(x \sqrt{\lambda}+\frac{1}{\sqrt{\lambda}} t\right)\right)}{A_{1} \sin \left(x \sqrt{\lambda}+\frac{1}{\sqrt{\lambda}} t\right)+A_{2} \cos \left(x \sqrt{\lambda}+\frac{1}{\sqrt{\lambda}} t\right)+\frac{\mu}{\lambda}} \\
\pm \frac{1}{2 \lambda} \frac{\sqrt{\mu^{2}-\lambda^{2}\left(A_{1}^{2}+A_{2}^{2}\right)}}{\left(A_{1} \sin \left(x \sqrt{\lambda}+\frac{1}{\sqrt{\lambda}} t\right)+A_{2} \cos \left(x \sqrt{\lambda}+\frac{1}{\sqrt{\lambda}} t\right)\right)+\mu}
\end{array}\right) \\
& u_{16,17}(x, t)=-\ln \left(\begin{array}{l}
-\frac{1}{2}-\frac{-\frac{1}{2}\left(A_{1} \cos \left(x \sqrt{\lambda}+\frac{1}{\sqrt{\lambda}} t\right)+A_{2} \sin \left(x \sqrt{\lambda}+\frac{1}{\sqrt{\lambda}} t\right)\right)}{A_{1} \sin \left(x \sqrt{\lambda}+\frac{1}{\sqrt{\lambda}} t\right)+A_{2} \cos \left(x \sqrt{\lambda}+\frac{1}{\sqrt{\lambda}} t\right)+\frac{\mu}{\lambda}} \\
\pm \frac{1}{2 \lambda} \frac{\sqrt{\mu^{2}-\lambda^{2}\left(A_{1}^{2}+A_{2}^{2}\right)}}{\left(A_{1} \sin \left(x \sqrt{\lambda}+\frac{1}{\sqrt{\lambda}} t\right)+A_{2} \cos \left(x \sqrt{\lambda}+\frac{1}{\sqrt{\lambda}} t\right)\right)+\mu}
\end{array}\right) \\
& u_{18}(x, t)=-\ln \left(-\frac{\mu^{2}-\lambda^{2}\left(A_{1}^{2}+A_{2}^{2}\right)}{\mu \lambda\left(A_{1} \sin \left(x \sqrt{\lambda}+\frac{-\rho \lambda^{2}+\mu^{2}}{\mu^{2} \sqrt{\lambda}} t\right)+A_{2} \cos \left(x \sqrt{\lambda}+\frac{-\rho \lambda^{2}+\mu^{2}}{\mu^{2} \sqrt{\lambda}} t\right)+\mu^{2}\right.}\right)
\end{aligned}
$$

As $A_{1}$ and $A_{2}$ are arbitrary constants, set $A_{1}=0, A_{2} \neq 0$ and $\mu=0$, solutions Eq. (65) to Eq. (66) turn out to the following periodic wave solutions:

$$
\begin{aligned}
& u_{19,20}(\xi)=-\ln \left(-\frac{1}{2}+\frac{1}{2} \tan \left(x \sqrt{\lambda}+\frac{1}{\sqrt{\lambda}} t\right) \pm \frac{1}{2} \sec \left(x \sqrt{\lambda}+\frac{1}{\sqrt{\lambda}} t\right)\right) \\
& u_{21,22}(\xi)=-\ln \left(-\frac{1}{2}-\frac{1}{2} \tan \left(x \sqrt{\lambda}+\frac{1}{\sqrt{\lambda}} t\right) \pm \frac{1}{2} \sec \left(x \sqrt{\lambda}+\frac{1}{\sqrt{\lambda}} t\right)\right)
\end{aligned}
$$

Alternatively, if we consider, $A_{1} \neq 0, A_{2}=0$ and $\mu=0$, solutions Eq. (65) - (66) become

$$
\begin{aligned}
& u_{23,24}(x, t)=-\ln \left(-\frac{1}{2}+\frac{1}{2} \cot \left(x \sqrt{\lambda}+\frac{1}{\sqrt{\lambda}} t\right) \pm \frac{1}{2} \csc \left(x \sqrt{\lambda}+\frac{1}{\sqrt{\lambda}} t\right)\right) \\
& u_{25,26}(x, t)=-\ln \left(-\frac{1}{2}-\frac{1}{2} \cot \left(x \sqrt{\lambda}+\frac{1}{\sqrt{\lambda}} t\right) \pm \frac{1}{2} \csc \left(x \sqrt{\lambda}+\frac{1}{\sqrt{\lambda}} t\right)\right)
\end{aligned}
$$

Case 3: When $\lambda=0$ (Rational function solutions)

Taking the same steps as for case- 1 and solving the system of equations yields sets of values of arbitrary constants as follows:

Set-7: $\left\{w=-\frac{2 \mu A_{2}-A_{1}^{2}}{\mu^{2}}, a_{0}=0, a_{1}=0, b_{1}=-\frac{2 \mu A_{2}-A_{1}^{2}}{\mu}\right\}$

Now substituting set-1 into Eq. (57), using the transformation $u=-\ln v$, we obtain abundant travelling wave solutions of Eq. (2) as follows:

$$
u_{27}(x, t)=-\ln \left(-\frac{4 A_{2}\left(2 \mu A_{2}-A_{1}^{2}\right)}{\mu\left(A_{1}^{2}\left(x+-\frac{2 \mu A_{2}-A_{1}^{2}}{\mu^{2}} t\right)^{2}+4 A_{2} A_{1}\left(x+-\frac{2 \mu A_{2}-A_{1}^{2}}{\mu^{2}} t\right)+4 A_{2}^{2}\right)}\right)
$$

Remark 1. All solutions have been checked by putting them back to the original Eq. (2) and found them correct.

\section{Graphical representations of some of the extracted solutions}

Graph is a powerful tool for describing the physical phenomena for the created any real-world problems. Therefore, we tend to create some graphs of obtained solutions. In order that, the obtained solutions are demonstrates the physical significance through with $3 \mathrm{D}$ and 2D graphics with the free choice of arbitrary parameters. The graphical illustrations of some extracted solutions are given in Figure.1-5 
with the assistance of MATLAB. Three dimensional and two-dimensional snapshots of the acquired solutions for the TDB equation shows the kink, singular kink and periodic behavior. Figure 1, and Figs. 3-4 represent the shape of kink and singular kink. On the other hand, Figure. 2 and Figure. 5 illustrates the periodic wave behaviors. The remaining of the obtained solutions represents the same physical phenomena.

(a)

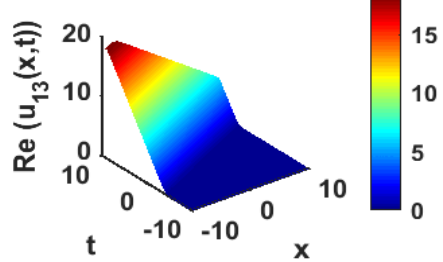

(c)

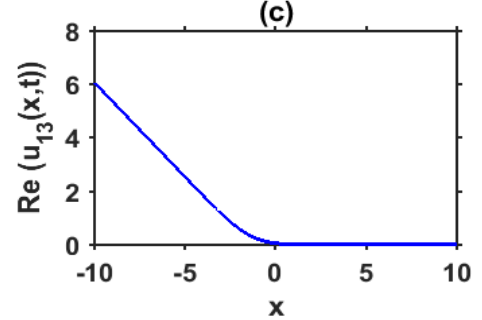

(b)

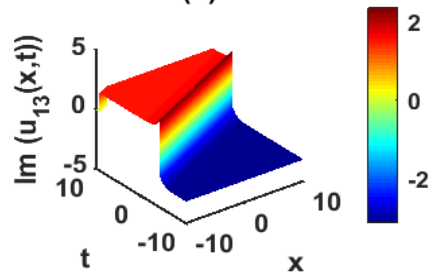

(d)

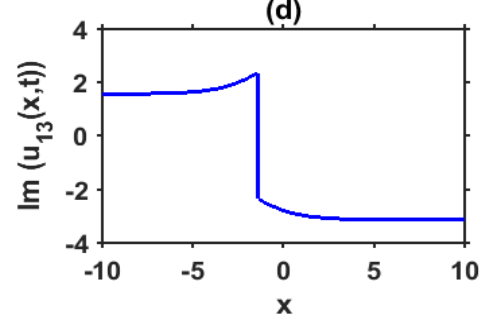

Fig. 1: (A)-(B): 3D Snapshots For The Solution The Solution Of TDB Equation Extracted By The Enhanced $(G$ '/ $G)$-Expansion Method For The Free Choice Of Arbitrary Parameters $A=1, \lambda=3, \mu=0.5$, And $\sigma=1$. (C)-(D): 2D Snapshots Of (A)-(B) At $t=0$.

(a)

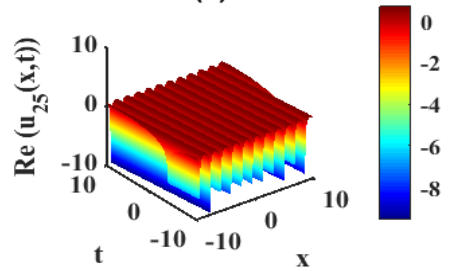

(c)

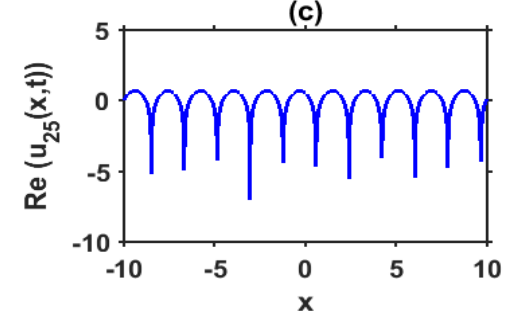

(b)

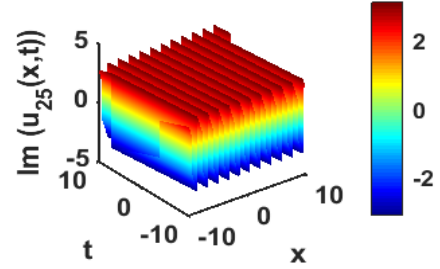

(d)

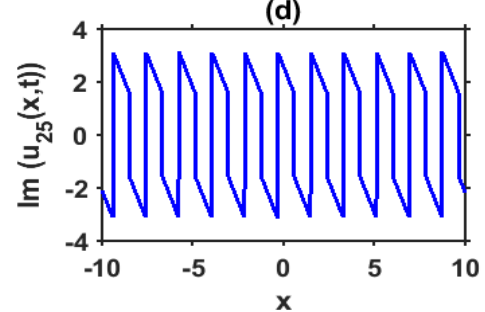

Fig. 2: (A)-(B): 3D Snapshots For The Solution The Solution Of TDB Equation Derived By The Enhanced $(G$ '/ $G)$-Expansion Method For The Free Choice Of Arbitrary Parameters $A=1, \lambda=1$, And $\mu=3$. (C)-(D): 2 D Snapshots Of (A)-(B) At $t=0$.

(a)

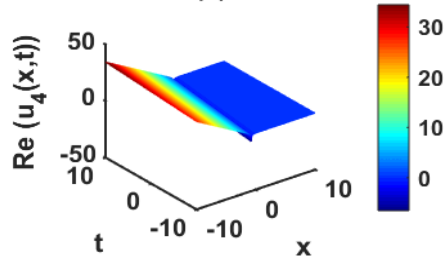

(c)

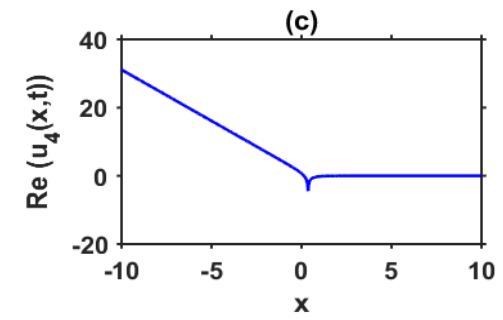

(b)

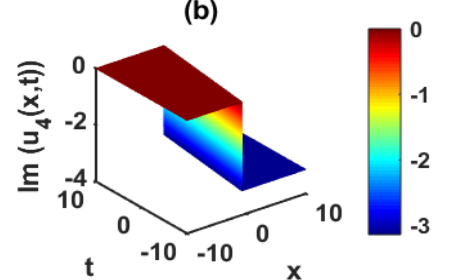

(d)

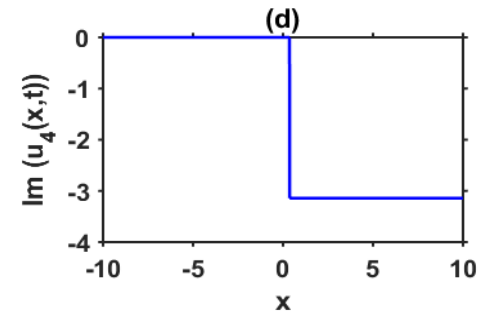

Fig. 3: (A)-(B): 3D Snapshots For The Solution The Solution Of TDB Equation Obtained By The Improved (1/ $\left.G^{\prime}\right)$-Expansion Method For The Parameters For The Free Choice Of Arbitrary Parameters $A=1, \lambda=3, \mu=1$, And $m=1$. (C)-(D): $2 \mathrm{D}$ Snapshots Of (A)-(B) At $t=0$. 
(a)

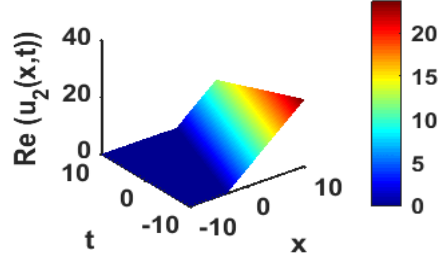

(c)

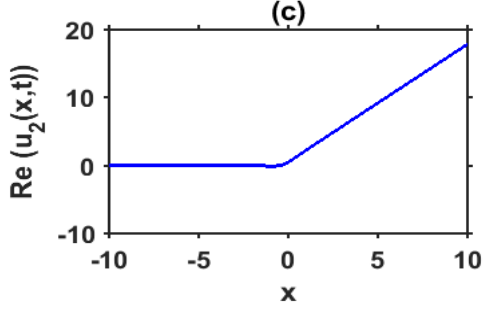

(b)

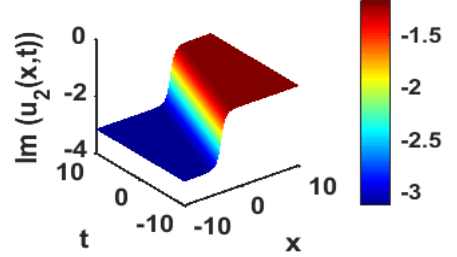

(d)

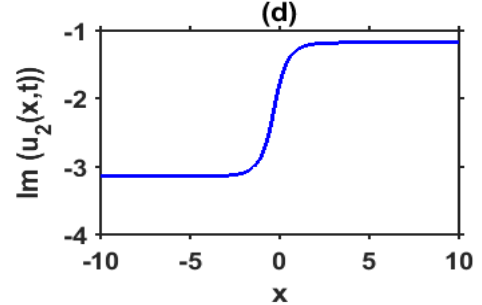

Fig. 4: (A)-(B): 3D Snapshots For The Solution The Solution Of TDB Equation Produced By The $(G / G, 1 / G)$-Expansion Method For The Parameters For The Free Choice Of Arbitrary Parameters $A_{1}=1, A_{2}=2, \lambda=-3, \mu=2$, And $m=1$. (C)-(D): 2D Snapshots Of (A)-(B) At $t=0$.

(a)

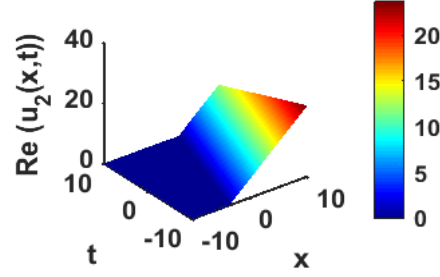

(c)

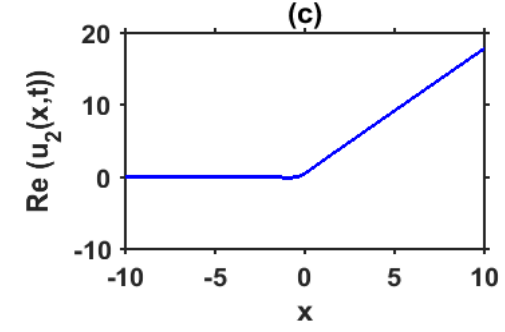

(a)

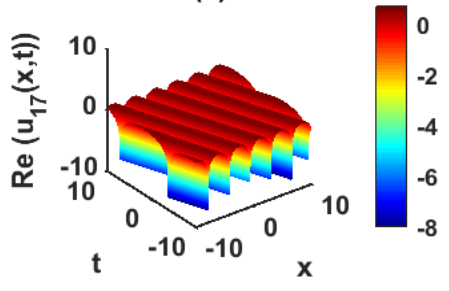

(c)

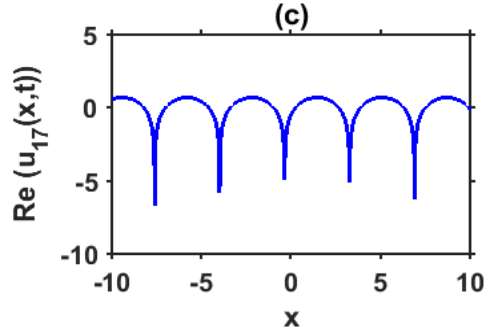

(b)

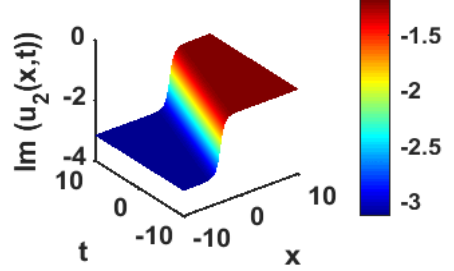

(d)

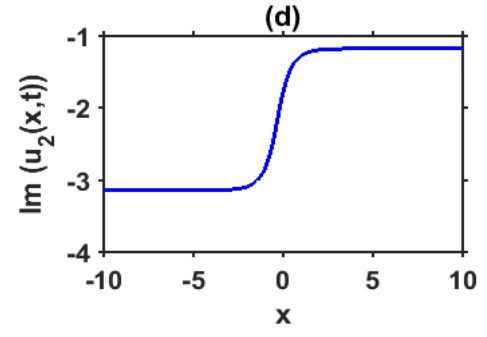

(b)

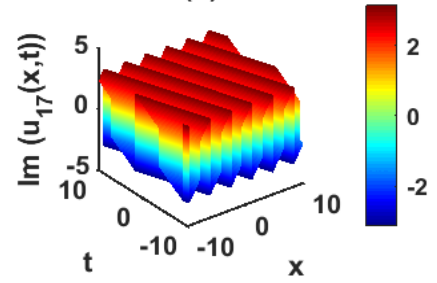

(d)

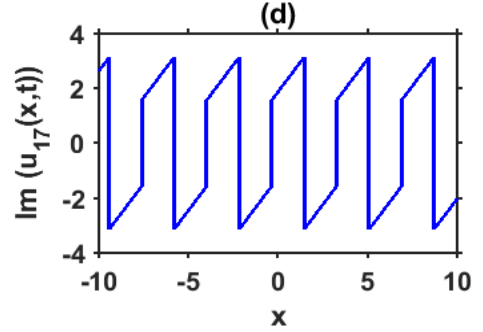

Fig. 5: (A)-(B): 3D Snapshots For The Solution The Solution Of TDB Equation Produced By The $\left(G^{\prime} / G, 1 / G\right)$-Expansion Method For The Parameters For The Free Choice Of Arbitrary Parameters $A_{1}=2, A_{2}=1, \lambda=3, \mu=1$, And $m=1$. (C)-(D): 2D Snapshots Of (A)-(B) At $t=0$.

\section{Results and discussion}

In this article, the derived solution for the TDB equation is a plentiful move by means of three different approaches. Here, our derived solutions have three forms referred to as hyperbolic function, trigonometric function and rational function solutions. Some solutions of the TDB equation are new and some solutions coincide the referred literature [33, 34]. The thinking about of assuming solutions and the auxiliary equation are completely extensive from $[33,34]$ and we also claim that some of the acquired solutions have not been reported in the past. Using the enhanced $\left(G^{\prime} / G\right)$-expansion method, we derived eighteen hyperbolic function solutions and eighteen trigonometric function solutions for TDB. In the improved $\left(1 / G^{\prime}\right)$-expansion method, we constructed seven new solutions which are not cited by the 
past researches. In the $\left(G^{\prime} / G, 1 / G\right)$-expansion method, we received the not only hyperbolic, trigonometric and rational solutions but also linear combination of the hyperbolic and trigonometric function solutions which are generally new formation.

From our observation, if we set $b_{i}=0, \lambda=0$ and negative the exponents of $\left(G^{\prime} / G\right)$ are zero in Eq. (6), then the executed method turn into the basic $\left(G^{\prime} / G\right)$ expansion method which was introduced by Wang et al. [21].Again if we substitute $m=0$ in Eq. (11) and omit the portion $\sum_{i=1}^{n} b_{i}(m+F(\xi))^{-i}$ then the improved $\left(1 / G^{\prime}\right)$-expansion method becomes the basic $\left(1 / G^{\prime}\right)$-expansion method which was introduced by Yokus[24]. Similarly, if we set $\mu=0$ in Eq. (16) and $b_{i}=0$ in Eq. (25) the $\left(G^{\prime} / G, 1 / G\right)$-expansion method reduces to the basic $\left(G^{\prime} / G\right)$ expansion method. Hence, the basic $\left(G^{\prime} / G\right)$ and $\left(1 / G^{\prime}\right)$-expansion method is only the particular case of our respective method. So, our derived solutions are quite different. In this regard, it can be shown that the obtained solution of TDB equation of Abazari[33] and Borhanifar and Moghanlu [34], is only the particular case of the enhanced $\left(G^{\prime} / G\right)$ and the $\left(G^{\prime} / G, 1 / G\right)$-expansion method. Therefore, in this paper we found some new solutions.

\section{Conclusion}

In this work, we obtained the large number of closed form soliton solutions of the Tzitzeica Dodd Bullough (TDB) equation by applying the enhanced $\left(G^{\prime} / G\right)$-expansion method, the improved $\left(1 / G^{\prime}\right)$-expansion method and the $\left(G^{\prime} / G, 1 / G\right)$-expansion method. The extracted solutions are different categories and more general from the previously obtained solutions which were presented by the other authors in the literature [33-34]. We observe that our results can be potentially found useful for applications in the field of nonlinear optics. All solutions are verified with the aid of maple packet program. The soliton solutions are expressed in terms of hyperbolic functions, trigonometric functions and rational functions. Therefore, it is shown that the enhanced $\left(G^{\prime} / G\right)$-expansion method, the improved $\left(1 / G^{\prime}\right)$ expansion method and the $\left(G^{\prime} / G, 1 / G\right)$-expansion method provides a very effective and powerful mathematical tool for solving nonlinear equations in mathematical physics and nonlinear optics.

\section{References}

[1] Debnath, L., Nonlinear partial differential equations for scientists and engineers, Springer Science \& Business Media, 2011. https://doi.org/10.1007/978-0-8176-8265-1.

[2] Whitham, G. B., Linear and nonlinear waves. Vol. 42. John Wiley \& Sons, 2011.

[3] Wazwaz, A. M., Partial differential equations and solitary waves theory. Springer Science \& Business Media, 2010. https://doi.org/10.1007/978-3642-00251-9.

[4] Wang, M., Zhou, Y. \& Li, Z., Application of a homogeneous balance method to exact solutions of nonlinear equations in mathematical physics. Physics Letters A, 216 (1-5), 1996, 67-75. https://doi.org/10.1016/0375-9601(96)00283-6.

[5] Wazwaz, A. M., Multiple-soliton solutions for the KP equation by Hirota's bilinear method and by the tanh-coth method. Applied Mathematics and Computation, 190 (1) (2007) 633-640. https://doi.org/10.1016/j.amc.2007.01.056.

[6] Fu, X., Cen, S., Li, C. F. \& Chen, X., Analytical trial function method for development of new 8-node plane element based on the variational principle containing Airy stress function. Engineering Computations, 27 (4), 2010, 442-463. https://doi.org/10.1108/02644401011044568.

[7] Malfliet, W., Solitary wave solutions of nonlinear wave equations. American Journal of Physics, 60 (7) (1992) 650-654. https://doi.org/10.1119/1.17120.

[8] Chow, K. W., A class of exact, periodic solutions of nonlinear envelope equations. Journal of Mathematical Physics, 36 (8) (1995) $4125-4137$. https://doi.org/10.1063/1.530951

[9] Fan, E., Multiple travelling wave solutions of nonlinear evolution equations using a unified algebraic method. Journal of Physics A: Mathematical and General, 35(32) (2002) 6853. https://doi.org/10.1088/0305-4470/35/32/306

[10] Fan, E., Extended tanh-function method and its applications to nonlinear equations. Physics Letters A, 277(4) (2000) 212-218. https://doi.org/10.1016/S0375-9601(00)00725-8.

[11] El-Wakil, S. A., \& Abdou, M. A., New exact travelling wave solutions using modified extended tanh-function method. Chaos, Solitons \& Fractals, 31(4) ( 2007) 840-852. https://doi.org/10.1016/j.chaos.2005.10.032.

[12] Bai, C., Exact solutions for nonlinear partial differential equation: a new approach. Physics Letters A, 288 (3) (2001) 191-195. https://doi.org/10.1016/S0375-9601(01)00522-9.

[13] Yusufoğlu, E., \& Bekir, A., Solitons and periodic solutions of coupled nonlinear evolution equations by using the sine-cosine method. International Journal of Computer Mathematics, 83 (12) ( 2006) 915-924. https://doi.org/10.1080/00207160601138756.

[14] Wu, J., \& Geng, X., Inverse scattering transform and soliton classification of the coupled modified Korteweg-de Vries equation. Communications in Nonlinear Science and Numerical Simulation, 53 (2017)83-93. https://doi.org/10.1016/j.cnsns.2017.03.022.

[15] Alquran, M. \& Jarrah, A., Jacobi elliptic function solutions for a two-mode KdV equation. Journal of King Saud University-Science, .31(4) (2017) 485-489. https://doi.org/10.1016/j.jksus.2017.06.010.

[16] Ebadian, A., Najafzadeh, S., Farahrooz, F. et al., On the convergence and numerical computation of two-dimensional fuzzy Volterra-Fredholm integral equation by the homotopy perturbation method. SeMA Journal, 75 (2018) 17-34. https://doi.org/10.1007/s40324-017-0109-1.

[17] Zhang, S., A generalized auxiliary equation method and its application to the (2+1)-dimensional KdV equations. Applied mathematics and computation,188(1) (2007)1-6. https://doi.org/10.1016/j.amc.2006.09.068.

[18] Hosseini, K., Ansari, R. \& Gholamin, P., Exact solutions of some nonlinear systems of partial differential equations by using the first integral method, Journal of Mathematical Analysis and Applications, 387(2) (2012) 807-814. https://doi.org/10.1016/j.jmaa.2011.09.044.

[19] Hosseini, K. Mayeli,P. \& Kumar, D., New exact solutions of the coupled sine-Gordon equation in nonlinear optics using the modified Kudryashov method. Journal of Modern Optics, 65(3) (2017) 361-364. https://doi.org/10.1080/09500340.2017.1380857.

[20] Khater, M. M. A. \& Kumar, D.,New exact solutions for the time fractional coupled Boussinesq-Burger equation and approximate long water wave equation in shallow water. Journal of Ocean Engineering and Science, 2(3) (2017) 223-228. https://doi.org/10.1016/j.joes.2017.07.001.

[21] Wang, M., Li, X., \& Zhang, J., The ( $\left.G^{\prime} / G\right)$-expansion method and travelling wave solutions of nonlinear evolution equations in mathematical physics. Physics Letters A, 372 (4) (2008) 417-423. https://doi.org/10.1016/j.physleta.2007.07.051.

[22] Hawlader, F. \& Kumar D., A variety of exact analytical solutions of extended shallow water wave equations via the improved ( $G^{\prime} / G$ )expansion method. International Journal of Physical Research, 5 (1) (2017) 21-27. https://doi.org/10.14419/ijpr.v5i1.7429. 
[23] Khan, K. \&Akbar M. A., Traveling wave solutions of nonlinear evolution equations via the enhanced ( $\left.G^{\prime} / G\right)$-expansion method. Journal of the Egyptian Mathematical Society, 22 (2) (2014) 220-226. https://doi.org/10.1016/j.joems.2013.07.009.

[24] Yokus A., Solutions of some nonlinear partial differential equations and comparison of their solutions, Ph.D. Thesis, Firat University, Turkey, 2011.

[25] Khater, M.M.A. \& Kumar, D., Implementation of three reliable methods for finding exact solution of $(2+1)$ dimensional generalized fractional evolution equations, Optical and quantum electronics. 50( 427) (2018). https://doi.org/10.1007/s11082-017-1249-3.

[26] Li, Lx., Li, Eq. \& Wang, Ml., The $\left(G^{\prime} / G, 1 / G\right)$-expansion method and its application to travelling wave solutions of the Zakharov equations. Applied Mathematics-A Journal of Chinese Universities, 25 (4) (2010) 454-462. https://doi.org/10.1007/s11766-010-2128-x.

[27] Mamun Miah, M., Shahadat Ali, H.M., Ali Akbar, M. et al., Some applications of the ( $\left.G^{\prime} / G_{a} 1 / G\right)$-expansion method to find new exact solutions of NLEEs. The European Physical Journal Plus, 132 (6) (2017) 252. https://doi.org/10.1140/epjp/i2017-11571-0.

[28] Wazwaz, A. M., The tanh method for travelling wave solutions to the Zhiber-Shabat equation and other related equations. Communications in Nonlinear Science and Numerical Simulation, 13(3) (2008) 584-592. https://doi.org/10.1016/j.cnsns.2006.06.014.

[29] Wazwaz, A. M., The tanh method: solitons and periodic solutions for the Dodd-Bullough-Mikhailov and the Tzitzeica-Dodd-Bullough equations. Chaos, Solitons \& Fractals, 25 (1) ( 2005) 55-63. https://doi.org/10.1016/j.chaos.2004.09.122.

[30] Khan, K., \& Akbar, M. A., Exact and solitary wave solutions for the Tzitzeica-Dodd-Bullough and the modified KdV-Zakharov-Kuznetsov equations using the modified simple equation method. Ain Shams Engineering Journal, 4(4), (2013) 903-909. https://doi.org/10.1016/j.asej.2013.01.010.

[31] Tang, Y., Xu,W., Shen, J. \& Gao, L. Bifurcations of traveling wave solutions for Zhiber-Shabat equation. Nonlinear Analysis: Theory, Methods \& Applications, 67(2) (2007) 648-656. https://doi.org/10.1016/j.na.2006.06.024.

[32] Wazzan, L., Solutions of Zhiber-Shabat and Related Equations Using a Modified tanh-coth Function Method. Journal of Applied Mathematics and Physics, 4(6) (2016) 1068-1079. https://doi.org/10.4236/jamp.2016.46111.

[33] Abazari, R., The (G/G)-expansion method for Tzitzéica type nonlinear evolution equations. Mathematical and Computer Modelling, 52(9) (2010) 1834-1845. https://doi.org/10.1016/j.mcm.2010.07.013.

[34] Borhanifar, A., \& Moghanlu, A. Z., Application of the ( $\left.G^{\prime} / G\right)$-expansion method for the Zhiber-Shabat equation and other related equations. Mathematical and Computer Modelling, 54(9) ( 2011) 2109-2116. https://doi.org/10.1016/i.mcm.2011.05.020.

[35] Manafian, J. \& Lakestani, M. Dispersive dark optical soliton with Tzitzéica type nonlinear evolution equations arising in nonlinear optics. Opt Quant Electron 48(116 ) (2016). https://doi.org/10.1007/s11082-016-0371-y.

[36] Islam, M. R., Application of the $\exp (-\$(\xi)$-expansion method for Tzitzeica type nonlinear evolution equations. Journal for Foundations and Applications of Physics, 4(1) (2017) 8-18.

[37] Hosseini, K., Bekir,A. \& Kaplan, M., New exact traveling wave solutions of the Tzitzéica-type evolution equations arising in non-linear optics. Journal of Modern Optics, 64(16) (2017) 1688-1692. https://doi.org/10.1080/09500340.2017.1302607.

[38] Inc, M., New type soliton solutions for the Zhiber-Shabat and related equations. Optik, 138 (2017) 1-7. https://doi.org/10.1016/j.ijleo.2017.02.103.

[39] Tala-Tebue, E., Djoufack, Z. I., Tsobgni-Fozap D. C., Kenfack-Jiotsa, A., Kapche-Tagne, F., \& Kofané,T. C., Traveling wave solutions along microtubules and in the Zhiber-Shabat equation. Chinese Journal of Physics, 55( 3) (2017) 939-946. https://doi.org/10.1016/j.cjph.2017.03.004.

[40] Kaplan, M., \& Hosseini K., Investigation of exact solutions for the Tzitzéica type equations in nonlinear optics. Optik, 154 (2018) $393-397$. https://doi.org/10.1016/j.ijleo.2017.08.116.

[41] He, J. H., \& Abdou M. A., New periodic solutions for nonlinear evolution equations using Exp-function method. Chaos, Solitons \& Fractals, 34 (5) ( 2007) 1421-1429. https://doi.org/10.1016/j.chaos.2006.05.072.

[42] Esen, A., Yagmurlu, N.M. \& Tasbozan, O., Double exp-function method for multisoliton solutions of the Tzitzeica-Dodd-Bullough equation. Acta Math. Appl. Sin. Engl. Ser, 32(2) (2016) 461-468. https://doi.org/10.1007/s10255-016-0572-y.

[43] Neirameh, A., New exact solitary wave solutions to the TDB and (2+1)-DZ equations. Palestine Journal of Mathematics, 4(2) (2015) 386-390.

[44] Hosseini, K., Ayati, Z. \& Ansari,R., New exact traveling wave solutions of the Tzitzéica type equations using a novel exponential rational function method. Optik, 148 (2017) 85-89. https://doi.org/10.1016/j.ijleo.2017.08.030.

[45] Kumar D., Hosseini K. \& Samadani F., The sine-Gordon expansion method to look for the traveling wave solutions of the Tzitzeica type equations in nonlinear optics, Optik, 149 (2017) 439-446. https://doi.org/10.1016/j.ijleo.2017.09.066.

[46] Hosseini, K., Ayati, Z. \& Ansari, R., New exact solutions of the Tzitzéica type equations arising in nonlinear optics using a modified version of the improved $\tan (\Phi(\xi) / 2) \tan ø(\Phi(\xi) / 2)$-expansion method. Opt Quant Electron 49(2730 (2017). https://doi.org/10.1007/s11082-017-1094-4

[47] Huber, A., A note on a class of solitary-like solutions of the Tzitzéica equation generated by a similarity reduction. Physica D: Nonlinear Phenomena, 237 (8) ( 2008) 1079-1087. https://doi.org/10.1016/j.physd.2007.12.008.

[48] He, B., Long Y., \& Rui W., New exact bounded travelling wave solutions for the Zhiber-Shabat equation. Nonlinear Analysis: Theory, Methods \& Applications, 71 (5) (2009) 1636-1648. https://doi.org/10.1016/j.na.2009.01.029. 